Ulrich A. Wien*

\title{
Flucht hinter den „Osmanischen Vorhang“. Glaubensflüchtlinge in Siebenbürgen
}

https://doi.org/10.1515/jemc-2019-2001

Abstract: The article deals with several periods and phenomena of migration to Transylvania behind the "Ottoman curtain" and its impacts between the first half of the sixteenth to the midst of the eighteenth century. In the fifteenth and sixteenth century the mental, political and confessional diverted or inhomogeneous frame conditions preordained the region as an area which was open minded for heterogeneous thinking, experiments and individuals or groups. Especially the dominance of the Ottoman Empire in the Balkans enabled adopting the reformation without Habsburg renitancy as a laboratory for religious heterogeneity. First, we notice that the later Reformer of Braşov (Johannes Honterus) imported the German Reformation to Transylvania after the end of his political exile in several centres of Reformation. After an expulsion order by the Habsburg King Ferdinand I, the Wittenberg minded reformer Paulus Wiener from Ljubljana (Slovenia) settled in Sibiu and became in 1553 the first superintendent and fortified the reform. Italian deviant preachers travelled through the realm of Queen Isabella Jagiellonica and King/Prince János II Zsigmond Szápolyai. After expulsion from Poland because of antitrinitarian ideas, the court physician Giorgio Biandrata tried to establish an open-minded protestant country. Freedom of preaching the gospel without hierarchical control - perhaps the aim of a Unitarian established regional church in the Principality - opened the border for antitrinitarian thinkers who had flown from Heidelberg, Italy and other parts of Europe. In the seventeenth century - in the 30 years' war - the Calvinist Gábor Bethlen founded an ambitious university Academy in Alba Iulia and offered resort to Calvinist professors of central Europe. At the same time (1622), the Diet of Transylvania provided refuge to Hutterites (handcrafters called Habaner) from Moravia to settle in Transylvania - interdicting mission. Their Anabaptist behaviour attracted 130 years later some of the "Transmigrants" who were expelled by the counterreformation minded Charles VI and Maria Theresia from Austrian, Styria and Carinthian underground Protestants. About 3000 persons were exact relocated to the "heretic corner" of the conquered province of Transylvania - the former

Article Note: Adolf Martin Ritter zum 23.11.2018 gewidmet.

*Corresponding author: Ulrich A. Wien, Institut für Evangelische Theologie,Universität KoblenzLandau, Landau 76829, Germany, E-mail: wien@uni-landau.de 
Ottoman vassal - where the Habsburgs had to respect the Basic Constitutional Law (by the Diploma Leopoldinum) including religious freedom of 1595 . The religiones receptae were Roman-catholic, Lutheran, Calvinist and Unitarian, but also the "tolerated" Rumanian-orthodox churches.

There has to be some research to the question of Ottoman-Christian interplay, motives and strategies of the heteronomy of the estates and the problem whether the non-absolutistic governance and policy was an advantage.

Keywords: humanist city reformation, Ottoman Empire, pioneer region of religious freedom, Transylvanian Antitrinitarism, Hutterites, Austrian Transmigrants, Frontier

Siebenbürgen (ung. Erdély, rum. Ardeal) war bereits im Spätmittelalter durch intellektuelle und ökonomische Verbindungen mit Zentraleuropa eng verwoben. Transferbeziehungen mit Süddeutschland, aber auch Schlesien und Kleinpolen wurden zwar im 16. Jahrhundert zeitweise beeinträchtigt, aber nie gänzlich unterbrochen. ${ }^{1}$

Die politische Gesamtlage führte allerdings zu einer spezifischen Sondersituation. Nach der für das ungarische Heer und die bis dahin führenden Magnatenfamilien verheerenden Niederlage gegen die Osmanen 1526 bei Mohács, hatten sich die Rahmenbedingungen grundlegend geändert. Das mittelalterliche Königreich Ungarn war untergegangen und zerfiel - in drei größere Einheiten. ${ }^{2}$

Exkurs: Vorgeschichte und Rahmenbedingungen

1 Konrad G. Gündisch, Siebenbürgen und die Siebenbürger Sachsen, Studienbuchreihe der Stiftung Ostdeutscher Kulturrat 8 (München: Langen Müller Verlag 1998), 58f; Harald Roth, Hermannstadt. Eine kleine Geschichte einer Stadt in Siebenbürgen. (Köln: Böhlau Verlag, 2006) 22f; ders., Kronstadt in Siebenbürgen. Eine kleine Stadtgeschichte (Köln: Böhlau Verlag, 2010), 54-56; Marco Bogade, „Memoria in der spätmittelalterlichen und frühneuzeitlichen bürgerlichen Sepulchralkultur von Hermannstadt und Nürnberg“, Zeitschrift für Siebenbürgische Landeskunde 39 (2016): 2-18 (2f).

2 Katalin Péter, Studies on the History of the Reformation in Hungary and Transylvania, hg. von Gabriella Erdélyi, Refo500 Academic Studies 45 (Göttingen: Vanderhoeck \& Ruprecht, 2018); Márta Fata, "The Kingdom of Hungary and Principality of Transylvania”, in A Companion to the Reformation in Central Europe, hg. von Howard Louthan und Graeme Murdock, vol. 61, Brill's Companions to the Christian Tradition (Leiden, Boston: Brill, 2015), 68-91; Gerald Volkmer, Siebenbürgen zwischen Habsburgermonarchie und Osmanischem Reich. Völkerrechtliche Stellung und Völkerrechtspraxis eines ostmitteleuropäischen Fürstentums 1541-1699, vol. 56, Schriften des Bundesinstituts für Kultur und Geschichte der Deutschen im östlichen Europa (München: De Gruyter, 2015), 28-42; Harald Roth, Kleine Geschichte Siebenbürgens (Köln: Böhlau Verlag ${ }^{4}$ 2012), 47-49; István Keul, Early Modern Religious Communities in East-Central Europe. Ethnic Diversity, Denominational Plurality, and Corporative Politics in the Principality of Transylvania 


\section{Disposition als „Fluchtraum“}

Siebenbürgen, im Karpatenbogen zwischen den Großmächten des Habsburgerreichs und des unter Suleiman dem Prächtigen expandierenden Osmanischen Reiches gelegen, hatte aufgrund der im 15. Jahrhundert geschlossenen politischen Union von drei politischen Ständen (der jeweiligen - in einem Rechtsraum privilegierten - „natio“ der ungarischen Magnaten, der quasiadligen Szekler und der privilegierten „Saxones“) eine Sonderentwicklung im Reich der Stefanskrone erfahren. Integrationsfaktor für die Zuwandernden waren im Rahmen der ökonomischen und politischen Opportunität die jeweiligen Gruppenrechte der jeweiligen „natio“. Der spezifische rechtliche Schutzraum, die ökonomische Prosperität und die religiöse (christliche) Konformität, die allerdings schon partiell Parallelgesellschaften von lateineuropäischer Katholizität und ostkirchlicher Orthodoxie kannte, bildeten die Basis der heterogenen Provinz-Gesellschaft(en). Die seit der Ansiedlung im 12. Jahrhundert auf „Königsboden“ (fundus regius) privilegierte Gruppe der „Saxones“ hatten in der 1486 gegründeten „Nationsuniversität“ ihre politische Vertretungskörperschaft. Filialsiedlungen auf Komitatsboden (mit Hörigengemeinden) wurden vom magyarischen Adel repräsentiert, partizipierten aber teilweise an religiösen Rechten (z. B. Pfarrwahlrecht der Gemeinden) der Saxones. Das Siedlungsgebiet dieser deutschsprachigen Gemeinden gehörte kirchenrechtlich unterschiedlichen Rechtsräumen an: Die Saxones mit der exemten Hermannstädter Propstei gehörten zum Erzbistum Gran (ung. Esztergom), die untertänigen Gemeinden zum Bistum Weißenburg (ung. Gyulafehérvár). Den Pfarrern stand auf Königsboden der ganze Zehnte zu; und die kirchlichen Kapitel waren zum Abgabenverband unter Leitung eines Generaldechanten zusammengeschlossen, der die Abgaben an die Vertreter der Krone übermittelte. Der Klerus war schon im 15. Jahrhundert größtenteils akademisch gebildet, das Konkubinat war immer noch die Regel.

Die Überlappungszone von habsburgischem und osmanischem Einflussgebiet war gekennzeichnet durch diverse Rechtsräume (sowohl in kirchlicher als auch politischer Hinsicht) mit differenzierten Rahmenbedingungen, also pluripolar strukturiert und ohne stringent durchgesetzte Option auf „Homogenität“ (intern) oder in der Gesamtregion, die sich ab 1541 zum Fürstentum

(1526-1691), vol. 143, Studies in Medieval and Reformation Traditions (Leiden: Brill, 2009), 3941 (map on p. 52); Zoltán Csepregi, „Konfessionsbildung und Einheitsbestrebungen im Königreich Ungarn zur Regierungszeit Ferdinands I“, Archiv für Reformationsgeschichte 94 (2003): 242-75; Márta Fata, Ungarn, das Reich der Stephanskrone, im Zeitalter der Reformation und Konfessionalisierung. Multiethnizität, Land und Konfession 1500-1700, vol. 60, Katholisches Leben und Kirchenreform im Zeitalter der Glaubensspaltung (Münster: Aschendorff, 2000), $16 \mathrm{f}$. 
konstituierte. Heterogenität herrschte auch in kirchlicher Hinsicht (partiell Episkopalrechte für den Hermannstädter Stadtpfarrer), aber auch aufgrund humanistisch geprägter Religiosität als Haltung und Grundkonstante, die sich auch in der Phase der Konfessionsbildung nach der Inkubationsphase der von Mitteleuropa ausstrahlenden Reformation erhielt. Das Szenario einer mittelfristigen Homogenisierung protestantischer Orientierungen war wegen theologischer Diffusität eingeschränkt und ist kurz- und mittelfristig misslungen. Es blieb ein humanistisch basierter, z. T. bewusst vermittlungstheologischer Schwebezustand (auch in den sich ausformenden theologischen Richtungen), also eine konfessionelle Unbestimmtheit bis nach der Wende zum 17. Jahrhundert erhalten. Das politische Interesse der Osmanen an einer diversifizierten Zersplitterung der Christenheit (und damit einer mangelnden machtpolitischen, antiosmanischen Opposition) in dem an der Peripherie ihres Herrschaftsraums gelegenen, tributpflichtigen, aber nicht direkt regierten Siebenbürgen (dar al ahd) war im 16. und 17. Jahrhundert erkennbar. Die Loyalität der Fürsten gegenüber den Osmanen inklusive des fürstlichen, politisch-ökonomischem Pragmatismus war wichtiger als die konfessionelle Orientierung.

Diese für Europa außergewöhnlichen Rahmenbedingungen prädestinierten Siebenbürgen als Laboratorium von Religionsdiskursen und zugleich als Fluchtraum für Individuen und Gruppen unterschiedlicher religiöser Herkunft und Orientierung.

\section{Entwicklung im 16. Jahrhundert}

Aufgrund einer klassischen Doppelwahl im November bzw. Dezember 1526 konkurrierten der Woiwode Johann I. Szapolyai und der Habsburger Ferdinand um den ungarischen Königsthron. ${ }^{3}$ Nach der Besetzung Budas 1541 konnte sich Siebenbürgen als zwar seit 1529 tributpflichtiges, aber innenpolitisch weitgehend autonomes Fürstentum etablieren: eine Ständemonarchie, die von drei privilegierten „nationes“ gemeinsam regiert und zusammen mit der fürstlichen Kanzlei verwaltet wurde. Das historische Siebenbürgen mit den sogenannten „Partes adnexae“ (offiziell gebräuchlicher Titel seit 1571: Regnum Transsylvaniae cum Partibus regnis Hungariae eidem adnexis) bildete den östlichen „Nachfolgestaat“ des mittelalterlichen Königreiches Ungarn. Die Mitte regierten die Osmanen als Paschalik direkt. Der Westen und Norden (königliches Ungarn) war von

3 Roth, Kleine Geschichte (wie Anm. 2), 48. 
den Habsburgern dominiert, und diese fielen - abgesehen von vorübergehenden Ausnahmephasen - als innersiebenbürgischer Machtfaktor praktisch aus. ${ }^{4}$

Siebenbürgen, das ab 1526 in den nächsten Jahrzehnten mehrfach von bürgerkriegsähnlichen Wirren geschüttelt wurde, entwickelte eine religiöse Sonderkultur: Es entwickelte sich zu einer Pionierregion der Religionsfreiheit. ${ }^{5}$ Der Landtag fixierte 1595 - über verschiedene Zwischenstufen - die Anerkennung des römisch-katholischen, lutherischen, calvinistischen und antitrinitarischen/ unitarischen Glaubens, und die rumänisch-orthodoxe Konfession wurde mehr oder weniger toleriert. ${ }^{6}$ Insofern hatte sich Osteuropa, speziell Siebenbürgen, von den Entwicklungen in Mitteleuropa abgekoppelt. Deswegen ist einerseits Thomas Maissen zuzustimmen, der feststellte: „Im Osten war dagegen ein konfessionelles Nebeneinander möglich, das jedoch nicht als Toleranz im modernen Sinne missverstanden werden sollte. Es beruhte darauf, dass hochadlige Herrschaftsträger ihresgleichen den politisch-religiösen Freiraum und die entsprechenden Verfügungsrechte nicht beschneiden wollten, den sie für sich selbst beanspruchten.“7 Für das Fürstentum Siebenbürgen mit seinen unterschiedlichen Rechtsräumen ist festzuhalten, dass innerhalb des Territoriums des Gesamtfürstentums und der Partes adnexae auf den Landtagen 1564 und 1568 zunächst Verkündigungsfreiheit gestattet wurde (die dem von Maissen

4 Volkmer, Stellung (wie Anm. 2), 113.

5 Ulrich A. Wien, „Religionsfreiheit in der Pionierregion Siebenbürgen“ in Siebenbürgen Pionierregion der Religionsfreiheit. Luther, Honterus und die Wirkungen der Reformation, vol. 15, ACADEMIA der Evangelischen Akademie Neppendorf (Bonn, Hermannstadt: Schiller Verlag, 2017), 7-16.

6 Edit Szegedi, „Zur Lutherrezeption in Siebenbürgen. Die Klausenburger Antitrinitarier und der Wittenberger Reformator im 16. Jahrhundert“ in Der Luthereffekt im östlichen Europa, hg. von Joachim Bahlcke, Beate Störtkuhl und Matthias Weber, vol. 64, Schriften des Bundesinstituts für Kultur und Geschichte der Deutschen im östlichen Europa (Berlin: De Gruyter, 2017), 63-70 (65); Ulrich A. Wien, „What does evangelical mean? On the complexity of disputes and religious debates between Wittenberg-, Swiss- and Unitarian-orientated groups in Transylvania" in Luther and Calvinism: Image and Reception of Martin Luther in the History and Theology of Calvinism, hg. von Herman J. Selderhuis, J. Marius J. Lange van Ravenswaay, vol. 42, Refo500 Academic Studies (Göttingen 2017), 355-78 (359); Ludwig Binder, Grundlagen und Formen der Toleranz in Siebenbürgen bis zur Mitte des 17. Jahrhunderts, vol. 11, Siebenbürgisches Archiv (Köln, Wien, 1976), 157.

7 Thomas Maissen, „Die Folgen der Glaubensspaltung für die politischen Strukturen in Europa“ in 500 Jahre Reformation. Rückblicke und Ausblicke, hg. von Peter Opitz (Berlin: De Gruyter, 2018), 181-200 (196); vgl. dazu die mythenkritische Position von Mihály Balázs, „Tolerant Country - Misunderstood Laws. Interpreting Sixteenth-Century Transylvanian Legislation Concerning Religion”, Hungarian Historical Review 2, no. 1 (2013): 85-108. 
beschriebenen Phänomen entspricht). Höchstwahrscheinlich ist folgendes Szenario: Die Meinungsführer (Franz Davidis und Giorgio Biandrata) strebten im Windschatten der Autorität des Regenten János II. Zsigmond Szapolyai (1540-1571) eine unitarische Landeskirche an. Dessen früher Tod verhinderte die Ausführung der Absichten. Das gegen die Unitarier gerichtete Innovationsverbot von 1572 verbunden mit einem Zensurgesetz des römisch-katholischen, aber gegenüber den Osmanen loyalen Regenten István Báthory (1571-1585) ${ }^{8}$ erreichte sein Ziel nur bedingt und sistierte die bis dahin erfolgten Entwicklungen. Andererseits gingen die gewissermaßen grundgesetzlichen Regelungen des Landtagsbeschlusses 1595 über die reine Verkündigungsfreiheit hinaus: Die zwischenzeitlich mit fürstlicher Unterstützung erfolgte Konsolidierung der protestantischen Bekenntnisgruppen inklusive der militärisch erzwungenen Anerkennung der römisch-katholischen Konfession proklamierte eine bis ins 19. Jahrhundert - trotz zeitweiliger Bevorzugung bzw. Benachteiligung - formal geltende Plurikonfessionalität, die dem im 16. Jahrhundert üblichen Prinzip territorialer religiöser Homogenität ${ }^{9}$ widersprach.

Hinter dem „osmanischen Vorhang“ (bis ca. 1688) bildete sich ein religiöses Refugium aus, das zum zeitweiligen oder länger währenden Auffangbecken von Religionsflüchtlingen oder devianter religiöser Einzelgänger bzw. Gruppen aus anderen Regionen Europas werden konnte. Im Gegensatz dazu verwies der Landtag mehrfach die von der katholischen Fürstenfamilie Báthory angeworbenen Jesuiten des Landes und untersagte die Wiederbesetzung der vakanten Bistümer Weißenburg und Großwardein. ${ }^{10}$

Sechs Aspekte der unterschiedlichen Mobilitäts- bzw. Migrationsbewegungen, die in Relation zu reformatorisch inspirierter Theologie stehen, sollen im Folgenden vorgestellt werden:

8 Fata, Kingdom (wie Anm. 2), 118.

9 Volker Leppin, „Toleranz im Horizont protestantischer Selbstverständigung in der Frühen Neuzeit“, In Schwierige Toleranz: der Umgang mit Andersdenkenden und Andersgläubigen in der Christentumsgeschichte, hg. von Mariano Delgado, Volker Leppin und David Neuhold, vol. 17, Studien zur christlichen Religions- und Kulturgeschichte (Fribourg, Stuttgart: Acad. Press/Kohlhammer, 2012), 81-90 (81).

10 Ulrich A. Wien, „Wirkungen des Calvinismus in Siebenbürgen im 16. und 17. Jahrhundert“, in Calvin und Calvinismus. Europäische Perspektiven, hg. von Irene Dingel und Herman J. Selderhuis, vol. 84, Veröffentlichungen des Instituts für Europäische Geschichte Mainz (Göttingen: Vandenhoeck \& Ruprecht, 2011), 127-153 (133f); jetzt erneut in Wien, Pionierregion (wie Anm. 5), 90-123 (97f). 


\subsection{Politischer Flüchtling Johannes Honterus wird Reformator Kronstadts}

Johannes Honterus (1498/99-1549), über den bis $\mathrm{zu}$ einer ersten Notiz des Humanisten Aventinus fast nichts sicher bekannt ist, musste wohl aus politischen Gründen seine Heimatstadt Kronstadt (ung. Brassó, rum. Braşov) verlassen. Aventin schildert ihn ,auf der Flucht“. ${ }^{11}$ Der später als Pädagoge, Gelehrte, Drucker und schließlich als Reformator Kronstadts bekannte Humanist Honterus war in seiner Heimatstadt - vermutlich aus politischen Gründen - nicht länger geduldet. Nürnberg (ab 22.10.1529), Krakau und Basel waren Stationen seiner Flucht/Exils zwischen 1529 und 1532. ${ }^{12}$ Er stellte 1532 eine Siebenbürgen-Karte in Basel her. Widmungsverse lassen darauf schließen, er habe ein Signal seines politischen Umschwenkens nach Hause gesandt. ${ }^{13}$ Jedenfalls konnte er wohl im Sommer 1533 in seine Heimatstadt zurückkehren. In der Zwischenzeit hatte der exilierte Intellektuelle allerdings ausreichend Gelegenheit, an seinen Aufenthaltsorten die reformatorischen Gedanken und Konkretionen kennen zu lernen. Zwar sind konkrete Schritte von ihm zu einer humanistisch profilierten Bildungsreform mit anschließender Stadtreformation in Kronstadt in Publikationen erst ab 1539 nachzuweisen. ${ }^{14}$ Es ist aber höchst wahrscheinlich, dass die

11 Johannes Turmair's genannt Aventinus Sämmtliche Werke, Band I. (München: Christian Kaiser, 1881) 652ff.; vgl. auch Jan Andrea Bernhard, Konsolidierung des reformierten Bekenntnisses im Reich der Stefanskrone. Ein Beitrag zur Kommunikationsgeschichte zwischen Ungarn und der Schweiz in der Frühen Neuzeit (1500-1700), vol. 19, Refo500 Academic Studies (Göttingen: Vandenhoeck \& Ruprecht, 2015/22017), 42.

12 Thomas Şindilariu, „Der Beginn der Reformation in Kronstadt - Ansätze zu einer Neubewertung “, in Quellen zur Geschichte der Stadt Kronstadt, Band VIII, Beiheft 2 Johannes Honterus. Reformatio ecclesiae Coronensis ac totius Barcensis provinciae. Corona 1543, hg. von Bernhard Heigl und Thomas Sindilariu. (Kronstadt 2017), 1-32 (3-9); Ludwig Binder, Johannes Honterus. Schriften, Briefe, Zeugnisse (Bukarest: Kriterion Verlag, 1996); Karl Reinerth, Die Gründung der evangelischen Kirchen in Siebenbürgen, vol. 5, Studia Transylvanica (Köln: Böhlau Verlag, 1979), 50-59; Oskar Wittstock, Johannes Honterus, der Siebenbürger Humanist und Reformator. Der Mann, das Werk, die Zeit (Göttingen: Vandenhoeck \& Ruprecht, 1970); Karl Kurt Klein, Der Humanist und Reformator Johannes Honter. Untersuchungen zur siebenbürgischen Geistes- und Reformationsgeschichte (Hermannstadt: Krafft \& Drotleff, a. g.; [etc., etc.], 1935); Oskar Netoliczka, Beiträge zur Geschichte des Johannes Honterus und seiner Schriften: Festgabe d. ev. Stadtpfarrgemeinde Kronstadt an den Landeskundeverein anläßlich der Vereinstage im Sept. 1930 (Kronstadt: Verlag des evang. Presbyteriums, 1930).

13 Erich Roth, Die Reformation in Siebenbürgen. Ihr Verhältnis zu Wittenberg und der Schweiz. Teil I: Der Durchbruch, vol. 2, Siebenbürgisches Archiv (Köln, Graz, 1962), 49-55.

14 Reinerth, Gründung (wie Anm. 12), 67f; Şindilariu, Beginn (wie Anm. 12), 15-18; Ulrich A. Wien, „Der Humanist Johannes Honterus“, in Johannes Honterus. Rudimenta Cosmographica Grundzüge einer Weltbeschreibung Corona/Kronstadt, 1542. Ins Deutsche, Rumänische und 
Nürnberger Vorbilder, ${ }^{15}$ aber wohl auch Einflüsse von Ökolampad von Honterus während seiner Flucht rezipiert und nach Siebenbürgen importiert wurden. ${ }^{16}$ Der politische Exilant importierte die Reformation.

\subsection{Strafdeportierter Paul Wiener wird Superintendent}

Der Laibacher Domherr und von König Ferdinand hoch geschätzte Finanzexperte Paul Wiener hatte sich in der humanistisch gesinnten und für die evangelische Theologie offenen Atmosphäre des Bistums Laibach (slowen. Ljubljana) unter anderem mit seinem Kollegen Primož Trubar für die evangelische Position exponiert - und als Witwer sogar wieder geheiratet. ${ }^{17}$ Aufgrund einer relativ spät erfolgten Anklage (1547) war er verfolgt und eingekerkert worden. Wohl wegen der besonderen persönlichen Beziehungen zu König Ferdinand blieb ihm ein Martyrium erspart. Er wurde nach Wien verlegt, verhört und erhielt die Gelegenheit zur Stellungnahme bzw. zum Widerruf mittels eines untergeschobenen Verhörprotokolls. Die Aufforderung zum Widerruf beantwortete er mit einer umfassenden Apologie „Inn causa fidei“, die auf 255 Seiten die Chancen, die

Ungarische übersetzte und kommentierte Faksimileausgabe, hg. von Robert Offner, Harald Roth, Thomas Sindilariu und Ulrich A. Wien. (Hermannstadt, Bonn: Schiller Verlag, 2015), 1137 (27).

15 Erich Roth, Reformation (wie Anm 13), 78-86; Ute Monika Schwob, Kulturelle Beziehungen zwischen Nürnberg und den Deutschen im Südosten im 14. bis 16.Jahrhundert, vol. 22, Buchreihe der Südostdeutschen Historischen Kommission (München: Oldenbourg, 1969), 82-140; Heinz Heltmann und Robert Offner, „Tiere und Pflanzen, Sozialkunde, Anatomie und Krankheitsnamen“, in Johannes Honterus. Rudimenta Cosmographica (wie Anm. 12), 67-91 (73f).

16 Bernhard, Bekenntnis (wie Anm. 10), $81 \mathrm{f}$.

17 Karl W. Schwarz, „Paul Wien(n)er“, in Biographisch-Bibliographisches Kirchenlexikon, Band 38 (Nordhausen: Verlag Traugott Bautz GmbH, 2017), Sp. 1517-1521; Reinerth, Gründung (wie Anm. 12), 190-208, der hier das Inhaltsreferat bietet seines Aufsatzes: Reinerth, „Das Glaubensbekenntnis Paul Wieners, des ersten evangelischen Bischofs der Siebenbürger Sachsen“, Archiv für Reformationsgeschichte 67 (1976): 203-31; Daniel Buda, „Hermannstadt/Sibiu - Paul Wiener“, in Europa reformata. European Reformation Cities and their Reformers, hg. von Michael Welker, Michael Beintker und Albert de Lange (Leipzig: Evangelische Verlagsanstalt, 2016), 209-216; Ulrich A. Wien, „Kein Platz für die Rechtfertigungslehre in Siebenbürgen?“ Konfluenzen - Jahrbuch der Abteilung Protestantische Theologie der Lucian Blaga Universität Sibiu 16/17 (2016-2017): 138-48 (143); Ders., „Pionierregion der Religionsfreiheit. Rezeption und Aushandlungsprozesse von Reformationen in Siebenbürgen im 16. Jahrhundert“, in Die Reformation im östlichen Mitteleuropa, hg. von Norbert Kersken (Marburg 2019 - Tagungen zur OstmitteleuropaForschung, im Druck). 
das Augsburger Interim bot, so weit als möglich ausnutzte, um eine nah an der Theologie Martin Luthers profilierte Stellungnahme vorzulegen. ${ }^{18}$ Durch Protektion König Ferdinands wurde er „begnadigt“ zum Landesverweis nach Siebenbürgen. Seine Zwangs-Verschickung hatte Folgen. Dem 1548 nach Hermannstadt (ung. Nagyszeben, rum. Sibiu) Verbannten „cuidam presbytero pauperi in captivitate detento“ wurde laut Bürgermeisterrechnung am 12. Juni 1549 ein erstes Almosen von zwei Gulden bewilligt. ${ }^{19}$ Die anfängliche Zurückhaltung des Hermannstädter Rates wich aber bald einer großen Zustimmung. Wiener wurde nicht nur vom Rat am 22. Juli 1549 als gut besoldeter Prediger bestellt, sondern am 11. Mai 1552 zum Hauptpfarrer berufen und am 6. Februar 1553 mit der Würde des ersten evangelischen Superintendenten ausgestattet. ${ }^{20}$ Seine profiliert an der Wittenbergischen Theologie orientierte Position hat die Hermannstädter und weitere siebenbürgische Theologen stark geprägt und über seinen Tod während der Pest am 16. August 1554 hinaus gewirkt (vor allem bei seinem Amtsnachfolger Matthias Hebler). Der Zwangsmigrant (Exulant) stabilisierte die Wittenbergische Reformation in Siebenbürgen und führte sie faktisch und kirchenrechtlich in die Unabhängigkeit. ${ }^{21}$

\subsection{Durchzugsstation italienischer Wanderprediger}

Etwa zeitgleich mit Wiener kam es zu einer ersten, temporären Begegnung Francesco Stancaros in Hermannstadt (ung. Nagyszeben, rum. Sibiu). Die 1549 entwickelte Idee einer Universitätsgründung in Siebenbürgen ließ sich allerdings nicht realisieren, und Stancaro zog 1551 weiter, womöglich auch deshalb, weil seine zunächst eher an der Schweizer Reformation orientierte Position keine Resonanz fand. ${ }^{22}$ Der wandernde Intellektuelle entwickelte subordinatianische

18 Abschrift des 16. Jahrhunderts in der Hamburger Staats- u. Universitätsbibliothek, Signatur Cod. Theol 1144; ÖNB, Signatur: Cod. 11,797; kritische Edition in Vorbereitung.

19 StA Hermannstadt, Bürgermeisterrechnung Martin Weiss, 1549, VII R.H.R. (Extradata provincialium et VII sedium).

20 Hermannstädter Kirchenbuch: „Paulus Wiener canonicus et concionator Laibacensis propter evangelii confessionem in Transilvaniam exulans ac per triennium Cibinii verbum Dei docens publico senatus stipendio in eiusdem ecclesiae patorem electus est XI. mensis Maii anno domini etc LII. Idem Paulus VI. die mensis februarii anno ... LIII. electus est omnibus parochorum suffragiis in superintendentem ...”; zit. nach Archiv des Vereins für siebenbürgische Landeskunde 11 (1872), 365f; vgl. Reinerth, Gründung (wie Anm. 12), $206 \mathrm{f}$.

21 Pikanterweise wurde der Kathedralzins 1555 trotz dieser „Autonomie“ an das Erzbistum Gran (ung. Esztergom) gezahlt.

22 Reinerth, Gründung (wie Anm. 12), 211-16; vgl. Adolf Schullerus, „Die Augustana in Siebenbürgen“, in Ders. Luthers Sprache in Siebenbürgen (Hermannstadt, 1923), 161-296 (168-84). 
Positionen. Erst bei seiner Rückkehr aus Königsberg 1553 fand er Unterstützung beim einflussreichen Adligen Péter Petrovics (ca. 1551-1557), dem er als Arzt diente. Durch dessen Protektion nahm er Einfluss auf die kontroverstheologischen Auseinandersetzungen in Siebenbürgen. Die Theologen, Synoden und der Landtag aber wiesen die schweizerisch-reformierte Theologie (der „Sakramentarier") noch zurück. ${ }^{23}$ Allerdings bereiteten seine Interventionen und Disputation der Rezeption der reformierten Theologie nachhaltig den Boden. ${ }^{24}$ Nachfolgende Zuwanderer konnten an diese Diskussionen ziemlich nahtlos anknüpfen.

\subsection{Rückzugsgebiet europäischer Antitrinitarier}

„The numbers of religious migrants grew after the 1550s-1560s as religious identity became more closely tied to political disputes. “25 Mit dem Ende des Trienter Konzils verschärften sich die theologischen Abgrenzungen weiter. Die humanistisch gesinnten, oft aus Italien stammenden Dogmenkritiker, die eine vernunftbasierte Theologie betrieben und das von Luther propagierte Schriftprinzip - sola scriptura - radikal, traditionskritisch und voraufklärerisch zuspitzten, suchten in einem biblizistisch begründeten, „historisch-ethischen Religionsmodell“ ${ }^{26}$ ihre Ideen $\mathrm{zu}$ bündeln. Nicht wenige verabschiedeten sich von der durch die spätantiken Ökumenischen Konzilien zur Glaubensnorm erhobenen Trinitätslehre und entwickelten Variationen antitrinitarischer

23 Philipp Melanchthons Gutachten von 1553 wurde bei Valentin Wagner in Kronstadt 1554 gedruckt (Philipp Melantho, de controversiis Stancari. Scripta anno MDLIII). Auch die - noch an Wittenberg orientierten - siebenbürgischen Theologen veröffentlichten Gegenschriften: Kaspar Helth (Confessio de mediatore generis humani Jesu Christo vero Deo et Homine, ... Vitebergae, anno 1555 ... Ex officina typographica Viti Creutzer) und Franz Davidis (Dialysis scripti Stancari ... Claudiopoli 1555). Die Synode positionierte sich am 13. Januar 1557 gegen ihn, und schließlich disputierte Davidis mit Stancaro am 31. Dezember 1557 vor Zeugen in Klausenburg, die Stancaro wegen seiner Abreise nicht zu Ende führte. Nach der Verurteilung von dessen Theologie auf der ungarischen Synode am 1. Mai 1558 publizierte Davidis eine letzte kontroverstheologische Schrift gegen Stancaro: Apologia adversus maledicentiam et calumnias Francisci Stancari, iussu et voluntate omnium docentium coelestem doctrinam incorrupte in ecclesiis Transilvanicis conscripta... . Claudiopoli 1558. Vgl. dazu Adolf Schullerus, Augustana (wie Anm. 22), 169-77.

24 Wien, Disputes (wie Anm. 6), $360 \mathrm{f}$.

25 Nicolas Terpstra, Religious Refugees in the Early Modern World. An Alternative History of the Reformation (New York: Cambridge UP, 2015), 115.

26 Kestutis Daugirdas, Die Anfänge des Sozinianismus. Genese und Eindringen des historischethischen Religionsmodells in den universitären Diskurs der Evangelischen in Europa, vol. 240, Veröffentlichungen des Instituts für Europäische Geschichte Mainz (Göttingen: Vandenhoeck \& Ruprecht, 2016). 
Vorstellungen. Diese wurden sowohl von römisch-katholischen als auch von fast allen bereits protestantischen Theologen abgelehnt und als Häresie politisch verfolgt. Giorgio Biandrata hatte als Hofarzt der 1551-1556 im polnischen Exil lebenden Königin Isabella Jagiellonica eine Vertrauensstellung innegehabt. Als er schließlich aus Polen flüchten musste, wandte sich der Antitrinitarier nach Weißenburg (ung. Gyulafehérvár, rum. Alba Iulia), um bei deren Sohn, dem erwählten ungarischen König/siebenbürgischen Fürsten János II. Zsigmond Szapolyai 1563 Aufnahme zu finden. ${ }^{27}$ Der junge, kränkliche und politisch eher schwache Regent gewährte dem humanistischen Mediziner weitgehenden Einfluss auf die Regierungs-, vor allem die Religionspolitik. ${ }^{28}$ Biandratas langfristiges Ziel war es wohl, über die Zwischenstation einer politischen Legitimation der reformiert-schweizerischen Theologie den Unitarismus als „Staatsreligion“ einzuführen. Auf dem Weg zum schließlich doch nicht erreichten Endstadium wurden - wie oben erwähnt - 1564 und 1568 die Bestimmungen der Verkündigungsfreiheit vom Landtag beschlossen. ${ }^{29}$ Als 1564 in einer konzertierten Aktion der Landtag eine dissimulierende Formulierung beschlossen hatte, die die erreichte konfessionelle Vielfalt (d.h. inklusive der schweizerisch-reformierten Richtung) rechtlich absicherte, wandte sich der Hofprediger, der sächsische Stadtpfarrer Franz Hertel/Davidis (ungarisch Dávid Ferenc) in Klausenburg (ung. Kolozsvár, rum. Cluj) mit Biandratas Protektion bereits 1565 antitrinitarischen Vorstellungen zu, die er im Januar 1566 öffentlich machte. ${ }^{30}$ Unterstützt

27 Mihály Balázs, „Franz Davidis“, in Radikale Reformation. Die Unitarier in Siebenbürgen, hg. von Ulrich A. Wien, Juliane Brandt, András Balogh, vol. 44, Studia Transylvana (Köln: Böhlau Verlag, 2013): 55-89 (70); vgl. dazu auch Mihály Balázs und Gizella Keserü, „Der siebenbürgische Unitarismus. Zum Forschungsstand“, in Radikale Reformation, ebenda, 11-36; vgl. auch die weiteren Beiträge auf den ersten 300 Seiten dieses Bandes; Daugirdas: Religionsmodell (wie Anm. 23), 165-76.

28 Balázs, Davidis (wie Anm. 27), 70f; Reinerth, Gründung (wie Anm. 12), 278-89; Keul, Communities (wie Anm. 2), 106-11.

29 Die evangelischen Kirchenordnungen des XVI. Jahrhunderts, 24. Band, Siebenbürgen: Das Fürstentum Siebenbürgen. Das Rechtsgebiet und die Kirche der Siebenbürger Sachsen, bearbeitet von Martin Armgart unter Mitwirkung von Karin Meese (Tübingen: Mohr Siebeck, 2012), 76; Edit Szegedi, Von konfessioneller Unbestimmtheit zu konfessioneller Konfrontation (Manuskript, 2017); Ulrich A. Wien, „Abschied von der Trinitätstheologie? Zur Komplexität von Disputationen und Religionsgesprächen in Siebenbürgen“, in Zwischen theologischem Dissens und politischer Duldung. Religionsgespräche in der Frühen Neuzeit, hg. von Irene Dingel, Volker Leppin und Kathrin Paasch, vol. 121, Veröffentlichungen des Instituts für Europäische Geschichte Mainz (Göttingen: Vandenhoeck \& Ruprecht, 2018), 77-110 (82).

30 Reinerth, Gründung (wie Anm. 12); Fata, Ungarn (wie Anm. 2), 105; Szegedi, Lutherrezeption (wie Anm. 6), 66; Mihály Balázs, Ungarländische Antitrinitarier IV. Ferenc Dávid, vol. 26, Bibliotheca Dissidentium (Baden-Baden, Bouxviller, 2008), 28. 
von Biandrata kam es zu mehrfachen Interventionen und Disputationen. Der Landtag wiederholte 1568 seinen Beschluss zur Sicherung der Verkündigungsund Religionsfreiheit (,dass aller Orten die Prediger das Evangelium predigen, verkündigen, jeder nach seinem Verständnis, und wenn es die Gemeinde annehmen will, gut, wenn nicht, so soll sie niemand mit Gewalt zwingen [...] denn der Glaube ist Gottes Geschenk"). ${ }^{31}$ Schließlich ergriff auf der Disputation in Großwardein (ung. Nagyvárad, rum. Oradea) im November 1569 der Regent János II. Zsigmond Szapolyai eindeutig zu Gunsten der antitrinitarischen Bewegung das Wort. $^{32}$ Im Januar 1571 wurde die Religionspräferenz des Fürsten dezidiert in den Geltungsbereich der Verkündigungsfreiheit aufgenommen. ${ }^{33}$ Wie oben bereits angedeutet, ging es nicht um religiöse Toleranz im modernen Sinn. Hier wurde in Übereinstimmung mit dem von Davidis in seinem Traktat „De falsa et vera unius Dei ... cognitione“ entwickelten Konzept eine vom Regenten angestrebte, auch die Differenzen zur Habsburgerdynastie markierende einheitliche Grundhaltung der Reformgesinnten gefördert: den Diskurs biblizistisch und ohne Rücksicht auf die dogmengeschichtlichen Entscheidungen der Antike zu begründen und darin die formale Einheit der Kirchenorganisation zu wahren. ${ }^{34}$

In der vom Massenphänomen religiöser Flüchtlinge ${ }^{35}$ gekennzeichneten Epoche wirkten weitere antitrinitarisch gesinnte Glaubensflüchtlinge zusammen mit Biandrata und Franz Davidis in Siebenbürgen. Mancherorts in Zentraleuropa hatte sich das Gerücht verbreitet, Siebenbürgen sei bereits ein antitrinitarisch

31 EKO 24, Siebenbürgen (wie Anm. 29), 84. Hierher gehört auch die Castellio-Rezeption durch die siebenbürgischen Antitrinitarier, vgl. dazu Balázs (wie Anm. 27); vgl. ebenfalls dazu Barbara Mahlmann-Bauer, „Glaubenskonformismus und Macht. Castellios Anthropologie der Verfolgung“, in Dies. (Hg.), Sebastian Castellio (1515-1563) - Dissidenz und Toleranz. Beiträge zu einer internationalen Tagung auf dem Monte Verità in Ascona 2015, vol. 46, Refo500 Academic Studies (Göttingen: Vandenhoeck \& Ruprecht, 2018), 489-523; vergleiche dazu ebenfalls Daniela Kohler, „Das Unkrautgleichnis (Mt 13,24-30) und seine Bedeutung für religiöse Toleranz. Die Exegesen in De haereticis im Vergleich mit denjenigen Calvins, Bezas, Luthers und Bullingers“, in Barbara Mahlmann-Bauer (Hg.), Sebastian Castellio (1515-1563) - Dissidenz und Toleranz. Beiträge zu einer internationalen Tagung auf dem Monte Verità in Ascona 2015, vol. 46, Refo500 Academic Studies (Göttingen: Vandenhoeck \& Ruprecht, 2018), 251-68 (266).

32 Wien, Disputes (wie Anm. 6); ders., Abschied (wie Anm. 29), 100-10.

33 EKO 24, Siebenbürgen (wie Anm. 29), 86.

34 Mihály Balázs, "Antitrinitarism", in A Companion to the Reformation in Central Europe, hg. von Howard Louthan und Graeme Murdock, vol. 61, Brill's Companions to the Christian Tradition (Leiden, Boston: Brill, 2015), 171-94 (182).

35 Terpstra, Refugees (wie Anm. 25), 5: „Even a mass phenomenon, religious exile had an individual face“. 
orientiertes Fürstentum geworden. ${ }^{36}$ Dort versammelten sich nun entsprechend gesinnte Glaubensflüchtlinge wie beispielsweise Jacobus Paläologus (ursprünglicher Dominikaner aus Chios), ${ }^{37}$ Johannes Sommer (aus Pirna), ${ }^{38}$ Matthias VeheGlirius (Kaiserslautern/Heidelberger Antitrinitarier), ${ }^{39}$ Christian Francken $^{40}$ sowie Fausto Socini. ${ }^{41}$ Aus der Gruppe der Heidelberger Antitrinitarier war Johannes Sylvanus 1572 wegen des Vorwurfs des Hochverrats in Heidelberg geköpft und verbrannt worden. ${ }^{42}$ Der ebenfalls dieser Gruppe angehörende, des Hochverrats verdächtige, vor der Verhaftung gewarnte Adam Neuser ${ }^{43}$ flüchtete und suchte sich nach einer anderthalbjährigen Irrfahrt publizistisch zu rechtfertigen. Von Siebenbürgen kommend beabsichtigte er, seine Rechtfertigung bei einer Wanderdruckerei im osmanisch besetzten Ungarn drucken zu lassen, weil er als

36 Martin Mulsow, „Der Haupttäter entkam“, in Madrasa of TIME, 4. (https://madrasaoftime. wordpress.com/2011/04/28/der-haupttater-entkam/, letzter Zugriff 5.9.2018); vgl. auch Terpstra, Refugees (wie Anm. 25), 114, 119.

37 Martin Rothkegel, „Iacobus Palaeologus und die Reformation. Antireformatorische Polemik in der Schrift Pro Serveto contra Calvinum“, in Ulrich A. Wien et al. (Hgg.), Radikale Reformation (wie Anm. 27), 91-134.

38 Lore Poelchau, De clade Moldavica elegiae XV. Vita Jacobi Basilici Heraclidis, vol. 8, Bibliotheca neolatina (Heidelberg: Manutius, 2001), 10-16.

39 Balázs, Ungarländische Antitrinitarier IV (wie Anm. 30), 38-41; Christopher J. Burchill, The Heidelberg Antitrinitarians, vol. 11, Bibliotheca Dissidentium (Baden-Baden, Bouxviller, 1989), 125-68; Robert Dán, Matthias Vehe-Glirius. Life and Work of a radical Antitrinitarian with his collected Writings, vol. 4, Studia Humanitatis (Leiden: Brill, 1982). Vehe nahm auch an den Diskussionen des von Biandrata zu Hilfe gerufenen Fausto Sozzini mit Franz Davidis teil („Et sane memini, cum ipso Francisco praesente Glirium eius symmystam et ex parte praeceptorem inter colloquendum urgerem, ut mihi diceret, an crederet Jesum Nasarenum iam revera esse Christum, illum nihil respondere voluisse, et sermonem nostrum praefracte alio detorsisse“, vgl. Dán, Vehe, 130).

40 József Simon, Die Religionsphilosophie Christian Franckens 1552-1610? Atheismus und radikale Reformation im Frühneuzeitlichen Ostmitteleuropa, vol. 117, Wolfenbütteler Forschungen (Wiesbaden: Harrassowitz, 2008).

41 Erich Wenneker, „Sozini (Socini, Sozzini) Fausto“, in Biographisch-Bibliographisches Kirchenlexikon 10 (Herzberg, 1995), Sp. 849-57; Dán, Vehe-Glirius (wie Anm. 39), 134-38.

42 Burchill, Heidelberg (wie Anm. 39), 16-84; Paul Philippi, „Sylvanus und Transsylvanien. Ein Stück Toleranzgeschichte zwischen Heidelberg und Siebenbürgen“, in Sechshundert Jahre Ruprecht-Karls-Universität Heidelberg 1386-1986, vol. 1, Semper Apertus (Heidelberg: Springer, 1985), 213-30.

43 Martin Mulsow, "Antitrinitarians and conversion to Islam. Adam Neuser reads Murad b. Abdullah in Ottoman Istanbul", in Conversion and Islam in the Early Modern Mediterranean. The Lure of the Other, hg. von Claire Norton. (London/New York: Routledge, 2017), 181-93 (Literaturverzeichnis!); ders., „Eine Konversion zum Islam. Adam Neuser (ca. 1530-1576)“, in Kriminelle, Freidenker, Alchemisten. Räume des Untergrunds in der Frühen Neuzeit (Köln: Böhlau Verlag, 2014), 33-59; Burchill, Heidelberg (wie Anm. 39), 85-123. 
Ausländer seine Darstellung in Siebenbürgen nicht drucken lassen konnte. Er fiel den Osmanen in Temeswar (ung. Temesvár, rum. Timişoara) in die Hände, wurde wegen Spionageverdachts verhaftet, ließ sich beschneiden und konvertierte zum Islam. Anschließend wurde er nach Konstantinopel verbracht und bekam den Namen Mustafa Beg. ${ }^{44}$ „It's fair to regard him as a religious refugee. ${ }^{45}$ Er korrespondierte weiterhin mit den Antitrinitariern in Siebenbürgen. ${ }^{46}$ Außerdem unterstützte er politisch - erfolglos - den Anspruch des antitrinitarisch gesinnten (aber habsburgtreuen) Kaspar Bekes auf den Fürstenthron in Siebenbürgen gegen den Gefolgsmann der Osmanen, den katholischen István Báthory. ${ }^{47}$ Bei einem Besuch des antitrintarischen Iacobus Palaeologus in Konstantinopel (türk. Istanbul) suchte Mustafa Beg diesen mithilfe eines Konvertitentraktats von Murad ibn Abdullah zur Konversion zum Islam zu bewegen, was dieser ablehnte. ${ }^{48}$

Matthias Vehe, den Johannes Sylvanus ${ }^{49}$ in Kaiserslautern geprägt hatte, war 1569/70 in die innere Emigration gegangen (übte wohl eine klandestine unitarische Religiosität), war 1576-79 Lehrer am unitarischen Kollegium in Klausenburg geworden und nannte sich Glirius. ${ }^{50}$ In Anlehnung an Palaeologus“ Konzeption verfasste er „Mattanjah“ und gilt als Inspirator der sabbatarischen Bewegung in Siebenbürgen. ${ }^{51}$ Für den in der Haft auf der Burg Deva 1579 verstorbenen Franz Davidis schrieb er eine „Defensio“. In Köln hatte er sich um 1574 dem Judentum zugewandt und erhielt eine Ausbildung bei Rabbinern. Der

44 Der Gelegenheitsdolmetscher und tragische Doppelagent suchte sich westlichen Besuchern als undercover-Agent zu präsentieren. Seine Konversion war aus Überzeugung, aber auch aus seinem Pragmatismus zu erklären. Er suchte eine Synthese von Islam und Antitrinitarismus mithilfe der Erforschung von in Konstantinopel zu findenden spätantiken christlichen Texten zu erreichen („Ex quibis [sic!] historiis videtur aparire Christianismum et Alcuranum non dissentire“, Mulsow, Antitrinitarians [wie Anm. 43], 190, Anmerkung 25) und arbeitete an der Übersetzung (von Teilen) des Qur'an ins Lateinische. Der schon für Heidelberg bezeugte Alkoholmissbrauch (Burchill, Heidelberg [wie Anm. 39], 10, 85, 89) verstärkte sich im Exil. Vgl. auch Mulsow, Haupttäter (wie Anm. 36), 6; Tobias P. Graf, The Sultan's Renegades. Christian-European Converts to Islam and the Making of the Ottoman Elite, 1575-1610 (Oxford: Oxford University Press, 2017), 82, 101, 149-55, 166, 197-201.

45 Graf, Renegades (wie Anm. 44), 82. Graf hält es aufgrund unzureichender Quellenbasis für unmöglich, eine seriöse zahlenmäßige Schätzung über die Flüchtlingszahlen in Istanbul abzugeben; immerhin zeigt die Grafik 4.1 ein deutsch-ungarisches Netzwerk mit circa 20 Personen; vgl. Graf, Renegades, 150.

46 Graf, Renegades (wie Anm. 44), 154, Fußnote 145.

47 Graf, Renegades (wie Anm. 44), 154.

48 Mulsow, Fluchträume (wie Anm. 42), 33-59, 49-51.

49 Burchill, Heidelberg (wie Anm. 39), 125; ebenda, 16-21.

50 Burchill, Heidelberg (wie Anm. 39), 125-68; Dán, Vehe-Glirius (wie Anm. 39), 133 f.

51 Balázs, Antitrinitarism (wie Anm. 37), 187; Burchill, Heidelberg (wie Anm. 39), 125-29. 
Jesuit Possevino beschrieb ihn als „Glirio tutto dato al Giudaismo“, ${ }^{52}$ dessen selbstgestecktes Ziel es war, eine antitrinitarische Übersetzung der Hebräischen Bibel $\mathrm{zu}$ erstellen. Mit seiner radikalen Theologie verstärkte er die zunehmend sich isolierende Haltung des nonadorantistisch eingestellten Franz Davidis. Nach seiner Entlassung 1579 aus dem Schulamt ${ }^{53}$ („lector scholae Claudiacae“54) in Klausenburg lebte er rund ein Jahrzehnt in Polen. Im Herbst 1589 nach Deutschland zurückgekehrt, wurde er sofort aufgespürt und verstarb nach fast einjähriger Haft im Verließ von Greetsiel 1590 und wurde, weil er „hesst geholden de besnidunge“ und „in velen anderen stücken den Joden gelick gewest, [...] in loco inhonesto begraven“. 55

\subsection{Aufnahme reformierter Professoren - die Akademie Weißenburg}

Während des 30-jährigen Krieges in Zentraleuropa wechselten mit dem jeweiligen Kriegserfolg auch die konfessionellen Vorgaben der Mächtigen für Untertanen und Personal. Die Universität Heidelberg wurde zunächst vorübergehend (1626-29) und dann 1632 für mehr als 22 Jahre geschlossen. ${ }^{56}$ Die reformierten siebenbürgischen Fürsten Gabriel Bethlen und Georg I. Rákoczi beteiligten sich partiell am Dreißigjährigen Krieg. Besonders Gabriel Bethlen ergriff 1628 die Chance, die in der Entwurzelung der reformierten Professoren lag und begründete in Weißenburg die Reformierte Akademie. Sie sollte den Rang einer international konkurrenzfähigen Hochschule erhalten. Friedrich Pauli, Jakob Kopisch und - insbesondere auch für dessen persönliche, individuelle Entwicklung entscheidend - Martin Opitz lehrten dort. ${ }^{57}$ Als erste waren die reformierten

52 Antonio Possevino, Transilvania, 1584, hg. von A. Veress (Budapest, 1913), 104, zitiert nach Dán, Vehe-Glirius (wie Anm. 39), 131. Dán (S136) zitiert die Einschätzung von Possevino: Transilvania, 136 und 139, „et pervertire le manifestissime profetie del testamento antico, stimolati da quel Glirio Giudaizante, et da altri [ ... ]“.

53 Dán, Vehe-Glirius (wie Anm. 39), 137.

54 Dán, Vehe-Glirius (wie Anm. 39), 131.

55 Dán, Vehe-Glirius (wie Anm. 39), 221.

56 Eike Wolgast, „Die Universität Heidelberg als Landesuniversität der Pfälzer“, in Weisheit und Wissenstransfer. Beiträge zur Bildungsgeschichte der Pfalz, hg. von Angelo Van Gorp und Ulrich A. Wien, vol. 1, Forschungen zur Pfälzischen Landesgeschichte (Ubstadt-Weiher: Verlag Regionalkultur, 2018), 29-42 (36f).

57 Achim Aurnhammer, „Tristia ex Transilvania. Martin Opitz’ Ovid-Imitatio im siebenbürgischen Exil (1622/23)“, in Deutschland und Ungarn in ihren Bildungs- und Wissenschaftsbeziehungen während der Renaissance, hg. von Wilhelm Kühlmann und Anton Schindling, vol. 62, Contubernium (Stuttgart: Franz Steiner Verlag, 2004), 253-72. 
Theologen Johann Heinrich Alsted (1588-1538), Johann Heinrich Bisterfeld (1605-1655) und Ludwig Piscator (ca. 1575-1656?) schließlich im Herbst 1629 dem Ruf nach Weißenburg gefolgt und verließen das kriegserschütterte nassauische Herborn im Westerwald. ${ }^{58}$ Alsted wurde Gründungsrektor, der mit seinen Kollegen gleich zu Beginn des Jahres 1630 die Aufbauarbeit begann. ${ }^{59}$ Die Akademie war mit jährlich 20.000 Gulden/Forint dotiert; adelige und nicht adelige Studierende wurden getrennt unterrichtet. Die Hochschule zog sogar ausländische Studenten aus Holland, Deutschland, Böhmen und Polen an. Weitere Schulen in Broos (ung. Szászváros, rum. Orăştie), Dej (ung. Dés), Neumarkt (ung. Marosvásárhély, rum. Târgu Mureş) und Klausenburg wurden materiell unterstützt. Schließlich unterrichtete sogar Jan Amos Komenský/Comenius (1592-1670) seit 1650 in Sárospatak und ab 1654 auch in Weißenburg. ${ }^{60}$ Trotz vereinzelter Kritik wurde die internationale Bildungsatmosphäre unter den ungarisch-reformierten Siebenbürgern sehr geschätzt, weil die Anstrengungen, den Bildungsstandard zu heben, als elementarer Baustein des ganzen religiösen Reformprozesses und der Stärkung der reformierten Kirche gegenüber den konfessionellen Rivalen angesehen wurde. ${ }^{61}$

In Weißenburg gelang es also, mithilfe von zum Teil geflüchteten, international renommierten Gelehrten, eine akademische Hochschule in Siebenbürgen zu gründen.

\subsection{Zufluchtsregion für Hutterer nach 1622}

Die Täufergemeinden waren 1536 in den habsburgischen Territorien fast ausgerottet worden. In Mähren konnten 1537 neue Täufergemeinden gegründet werden, wo es 1545 bereits 31 „Haushaben“ (Höfe mit bis zu 500 Einwohnern $^{62}$ )

58 Gerhard Menk, „Das Restitutionsedikt und die kalvinistische Wissenschaft. Die Berufung Johann Heinrich Alsteds, Philipp Ludwig Piscators und Johann Heinrich Bisterfelds nach Siebenbürgen“, in Ders., Zwischen Katheder und Kanzel. Protestantische Pfarrer- und Professorenprofile zwischen dem 16. und 20. Jahrhundert (Marburg: Jonas Verlag, 2011), 697-734, 722-34.

59 Menk, Restitutionsedikt (wie Anm. 58), 733.

60 Graeme Murdock, Calvinism on the Frontier 1600-1660. International Calvinism and the Reformed Church in Hungary and Transylvania (Oxford: Oxford University Press, 2000), 82-83 und 86-93; Menk, Restitutionsedikt (wie Anm. 58), 733.

61 Murdock, Calvinism (wie Anm. 60), 109.

62 Astrid von Schlachta, „Die Geschichte der Täufer in Tirol. Die Täufer im europäischen Kontext“, in Verbrannte Visionen? Erinnerungsorte der Täufer in Tirol, hg. von Astrid von 
gab. ${ }^{63}$ Besonders in Südmähren, wo Flüchtlingsgruppen aus Süddeutschland, Tirol und der Schweiz Zuflucht gefunden hatten, überlebten diese bis 1622. Sie lebten in Gütergemeinschaft, hatten Gemeinschaftsküchen und Refektorien, und Kinder wurden kollektiv erzogen. Weil die meisten Hutterer Handwerker waren, hatte der mährische Landtag die gnadenhalber Tolerierten als de facto steuerzahlende „ordentliche“ Bewohner akzeptiert. ${ }^{64}$ Der seit Januar 1622 in Mähren als kaiserlicher Gubernator amtierende Kardinal Dietrichstein gab den Befehl, die an der Wende zum 17. Jahrhundert auf 20000 Personen geschätzte Gruppe der Täufer des Landes zu verweisen, sofern sie nicht bekehrungswillig waren. Etwa ein Drittel der hutterischen Bevölkerung wählte die Auswanderung nach Oberungarn oder Siebenbürgen. ${ }^{65}$ Spätestens 1629 dürften die letzten nicht assimilierungswilligen Hutterer das Land verlassen haben. Ihnen hatte der siebenbürgischen Landtag 1622 - unter Vorbehalt des Missionsverbots - die ungehinderte Ausübung ihres Glaubens und freie Ausübung in allen Handwerken versprochen. Nach der Vertreibung aus Mähren zogen ca. 1200 Personen nach Unterwinz (ung. Alvinc, rum. Vinţu de Jos) in Siebenbürgen (nach); die Mortalität betrug $50 \%$. Weitere gleichgesinnte Gruppen folgten ihnen 1629, 1635, 1644 sowie $1649 .{ }^{66}$ Eine zum Teil zuvor schon bestehende Spezialisierung auf Töpferei und Fayence-Herstellung betrieben diese Gruppen weiter, deren Produkte als „Habaner“-Ware bekannt sind. ${ }^{67}$

Schlachta, Ellinor Forster, Giovanni Merola. (Innsbruck: Innsbruck University Press, 2007), 8-47 (29).

63 Thomas Winkelbauer, „Die Vertreibung der Hutterer aus Mähren 1622: Massenexodus oder Abzug der letzten Standhaften?“, in Glaubensflüchtlinge. Ursachen, Formen und Auswirkungen frühneuzeitlicher Konfessionsmigration in Europa, hg. von Joachim Bahlcke (Berlin: LIT, 2008), 207-33 (211); vgl. auch Astrid von Schlachta, „The Austrian Lands“, in A Companion to the Reformation in Central Europe, hg. von Howard Louthan und Graeme Murdock, vol. 61, Brill's Companions to the Christian Tradition (Leiden, Boston: Brill, 2015), 68-91.

64 Winkelbauer, Hutterer (wie Anm. 63), $212 \mathrm{f}$.

65 von Schlachta, Täufer (wie Anm. 62), 40f: dies., The Austrian Lands (wie Anm. 63), 85; Heinold Fast, „Hutterische Brüder“ in Theologische Realenzyklopädie, Band 15 (Berlin/New York: De Gruyter, 1986), 752-56 (754); James M. Stayer, „Täufer/Täuferische Gemeinschaften“, in Theologische Realenzyklopädie, Band 32 (Berlin/New York: De Gruyter, 2001), 597-617 (605f, 613).

66 Winkelbauer, Hutterer (wie Anm. 63), 219.

67 von Schlachta, Täufer (wie Anm. 62), 36-38. Siehe Abbildungen 1 und 2 von Habaner Töpferware aus dem Siebenbürgischen Museum (Gundelsheim/Neckar). 


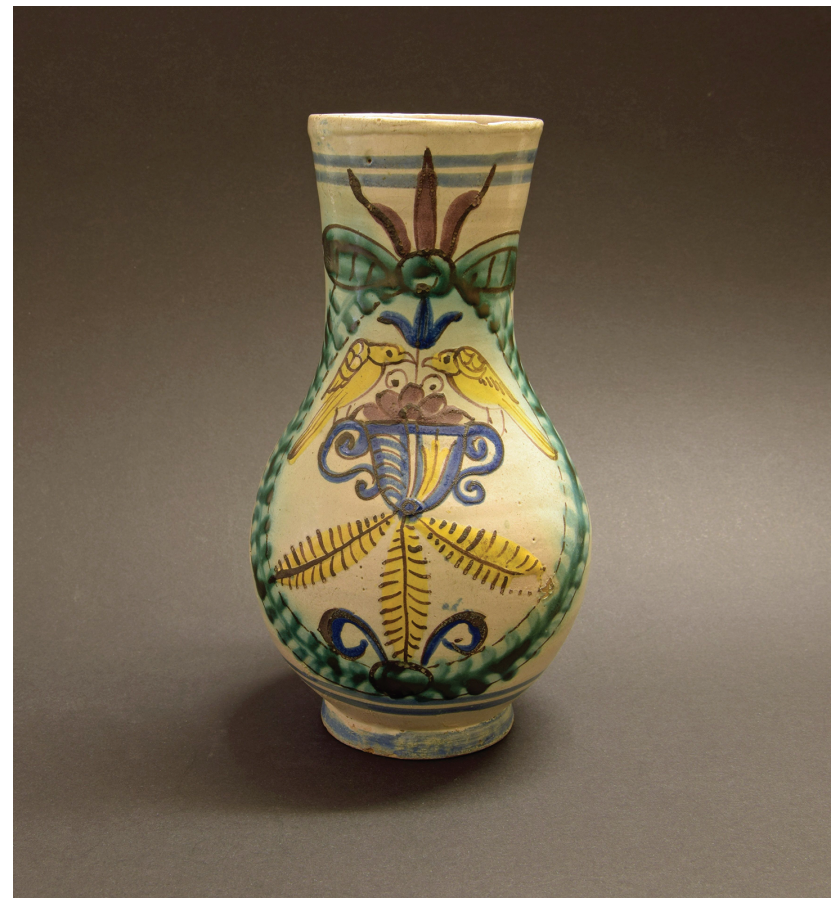

Abbildung 1: Habaner Krug H 20, Rd 7, Bd 6,4, D max. 11,3 (1759), Halbfayence, bemalt. Inventarnummer: SMG 05623. Foto: Dr Markus Lörz.

In Großschützen (Slowakei) und Unterwinz wurde 1667-1695 die Gütergemeinschaft aufgegeben. Die auf wenige Dutzend zusammen geschmolzenen Hutterer von Unterwinz ${ }^{68}$ wurden von ursprünglich klandestinen Kärntner „Transmigranten“ verstärkt, die sich 1755 als Tagelöhner bei den fast in Auflösung begriffenen Hutterern in Unterwinz verdingt und sich ihnen angeschlossen hatten.$^{69}$ Die Konvertiten folgten den ursprünglichen Regeln. Die Siedlung in Unterwinz lösten die Habsburger Behörden auf und zerstreuten die Bevölkerung in Siebenbürgen. Dem politischen Verfolgungsdruck wichen die Hutterer koordiniert 1767 aus und wanderten heimlich über die Karpaten in die Walachei und 1770/71 nach Russland und

68 Buchinger, „Die Geschichte der Kärtner Hutterischen Brüder in Siebenbürgen und in der Walachei (1755-1770)“, in Rußland und Amerika. Ein Beitrag zum Schicksal von Kärntner Transmigranten und zur Geschichte der heutigen Hutterischen Bruderhöfe in den USA und Kanada, vol. 172, Carinthia. Zeitschrift für Geschichtliche Landeskunde von Kärnten (Klagenfurt: Verlag des Geschichtsvereins für Kärnten, 1982), 145-302 (155).

69 Buchinger (wie Anm. 68), 173-75, 180-236; von Schlachta, Hutterer (wie Anm. 62), 42. 


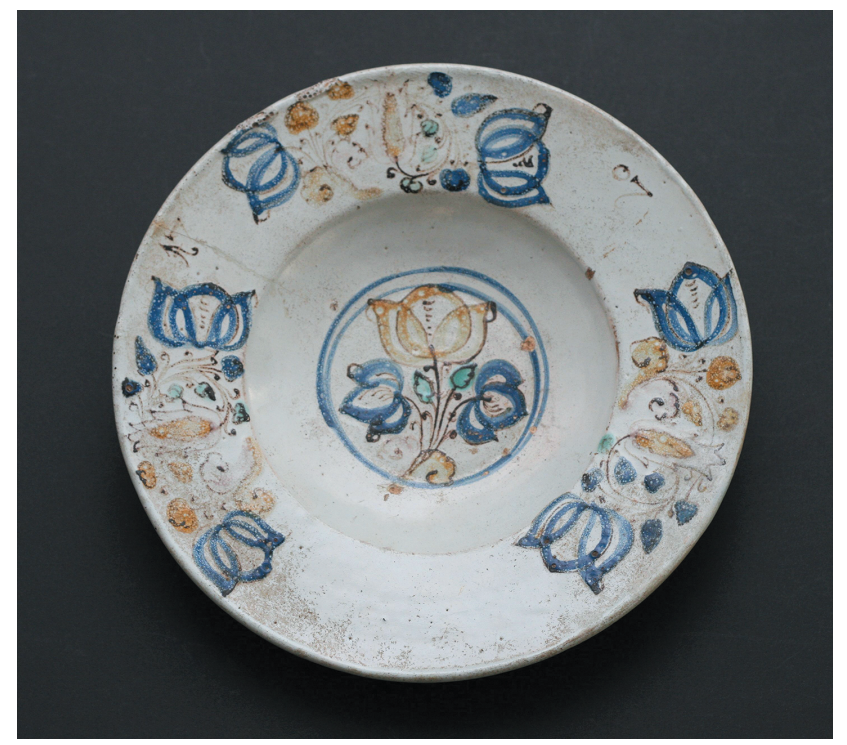

Abbildung 2: Habaner Teller H max. 6, min. 4, D 30,3 (1707), Halbfayence, bemalt. Inventarnummer: SMG 07753. Foto: Dr Markus Lörz.

in die heutige Ukraine weiter. Sie entzogen sich der dort 1874 auferlegten Militärpflicht durch Auswanderung in die USA und Kanada (mit 300 Bruderhöfen). ${ }^{70}$

\subsection{Nachwirkungen. Nach dem Fall des „osmanischen Vorhangs“: Zwangsverschickung im Habsburgerreich}

Die in drei Wellen zwischen 1734/37, 1752/57 und 1773/75 verfolgten oberösterreichischen, Kärntner und steirischen Geheimprotestanten wurden durch Kaiser Karl VI. und Kaiserin Maria Theresia - euphemistisch als „Transmigranten“ bezeichnet - zwar ebenfalls nach Siebenbürgen deportiert, aber nicht mehr hinter den „osmanischen Vorhang“. Denn die formale Anerkennung der Religionsgesetze Siebenbürgens inklusive der kirchlichen Unabhängigkeit durch die Habsburger im Diploma Leopoldinum $(1690 / 91)^{71}$ hatte dazu geführt, dass die

70 Buchinger (wie Anm. 68), 236-58; von Schlachta, Hutterer (wie Anm. 62), 42f; Winkelbauer, Hutterer (wie Anm. 63), 221.

71 Paul W. Roth, "Das Diploma Leopoldinum”, in Zsolt K. Lengyel, Ulrich A. Wien (Hg.), Siebenbürgen in der Habsburgermonarchie. Vom Leopoldinum bis zum Ausgleich (1690-1867), vol 34, Archiv für Siebenbürgische Landeskunde. (Köln: Böhlau Verlag, 1998), 1-11. 
Geheimprotestanten in diese multikonfessionell und -religiös geprägte Region zwangsumgesiedelt werden sollten. Unter dem Vorwand, sie seien Ketzer, politische Unruhestifter und Aufwiegler, wurden die kriminalisierten Evangelischen oft in ihren Eigentums- und Persönlichkeitsrechten beschnitten. Sie mussten das Land meist ohne den teilweise erst später erzielten Verkaufserlös ihres Hofes verlassen, nicht zu selten auch ihre Kinder unter 16 Jahren zurücklassen. In drei Zeitabschnitten sind diese sogenannten „Transmigranten“ allerdings aus religiösen Gründen zwangsdeportiert worden. Die kaiserliche Regierung hatte aber aus den Fehlern des Salzburger Bischofs von Firmian gelernt, der 20000 Evangelische aus seinem Land 1731/32 vertrieben hatte. Folglich entschloss sich Kaiser Karl VI. zur Zwangsumsiedlung der hartnäckigen evangelischen Bevölkerung innerhalb seines Reiches. Einerseits wollten er und die Landesherren rein katholische Gebiete regieren, um die absolutistische Macht auszubauen. Andererseits wollte der Kaiser die „Transmigranten“ aber als Steuerzahler behalten und zugleich das durch die verschiedenen Kriege entvölkerte Siebenbürgen durch einen Bevölkerungszufluss wirtschaftlich kräftigen. Außerdem gab es dort die von ihm $\mathrm{zu}$ duldenden „Akatholiken“, zu denen man die „Ketzer“ aus Österreich hinschicken konnte. ${ }^{72}$

In einer ersten Phase zwischen 1734 und 1737 kam es zur Umsiedlung von über 700 Evangelischen aus dem Salzkammergut und Kärnten, die über die Donau und Theiß verschifft wurden und schließlich zum großen Teil in den siebenbürgischen Dörfern Neppendorf (ung. Kistorony, rum. Turnişor) und Großau (ung. Kereszténysziget, rum. Christian) angesiedelt wurden.

In einer zweiten Phase unter der Kaiserin Maria Theresia wurden zwischen 1752 und 1757 vorwiegend aus Oberösterreich, Kärnten und aus der Steiermark (von dort sehr wenige) evangelische Familien umgesiedelt. Anfangs mussten sie ihre Kinder zurücklassen, ab 1755 wurden die Familien nicht mehr so häufig auseinandergerissen. Zwischen 1752 und 1757 gingen insgesamt 39 Transporte mit rund 3000 Personen ab, von denen ein erheblicher Teil in dem entvölkerten siebenbürgischen Dorf Großpold (ung. Nagyapold, rum. Apoldu de Sus) ${ }^{73}$

72 Der vorangehende Abschnitt fasst kompakt die neuesten Forschungsergebnisse zusammen; er basiert auf den Beiträgen von Stephan Steiner, Transmigration. Ansichten einer Zwangsgemeinschaft, S 331-60; Astrid von Schlachta, Die Emigration der Salzburger Kryptoprotestanten, 63-92; Rudolf K. Höfer, Geheimprotestantismus in der Steiermark, 93-122; Christine Tropper, Geheimprotestantismus in Kärnten, 123-54 und Andreas Hochmeir, Geheimprotestantismus im Land ob der Enns, 155-84, in dem Sammelband von Rudolf Leeb, Martin Scheutz, Dietmar Weikl (Hgg.): Geheimprotestantismus und evangelische Kirchen in der Habsburgermonarchie und im Erzstift Salzburg (17./18. Jahrhundert), vol. 51, Veröffentlichungen des Instituts für Österreichische Geschichtsforschung (Wien, München, 2009).

73 Mathias Beer, „Die Landler. Ein Versuch eines geschichtlichen Überblicks“, in Die siebenbürgischen Landler, hg. von Martin Bottesch, Franz Grieshofer und Wilfried Schabus (Köln: 
angesiedelt wurde. ${ }^{74}$ Diese „Exulanten“ wurden schwer benachteiligt. Obwohl viele als wohlhabend gelten konnten, kamen die meisten praktisch mittellos in Siebenbürgen an. Dort aber erging es ihnen erst richtig schlecht. Der Leiter des „Transmigranten-Inspektorats“, Hofrat Martin Vankhel von Seeberg, verschleuderte durch Misswirtschaft einen Großteil der mit großer Verzögerung eintreffenden Verkaufserlöse des früheren Besitzes der Deportierten. Er baute einen kasernenartigen Wohnblock, das „Retranchement“ in Hermannstadt, in dem die zwangsumgesiedelten Bauern jeweils in einem Zimmer wohnen sollten. Obwohl sie meist keine Handwerker waren, sollten sie dort auch ihre Werkstätten haben. Binnen des ersten Jahres verstarben fast $40 \%$ der Deportierten, teils noch auf dem - vom ersten bis zum letzten Tag militärisch gesicherten - Transport (auf Schiffen über die Drau und Donau sowie auf dem Landweg von Temeswar bis Hermannstadt) oder unmittelbar nach der Ankunft. Dass die von den Politikern verharmlosend „Transmigration“ genannte Deportation für etwa ein Drittel der insgesamt Betroffenen gewissermaßen die Todesstrafe war, wurde von den Verantwortlichen billigend in Kauf genommen. Die älteren starben häufiger als die jüngeren Deportierten, vermutlich nicht nur wegen der Strapazen, sondern auch auf Grund der großen seelischen Belastungen.

Erst unter dem Nachfolger des abgesetzten von Seeberg, Hofkammerrat Freiherr Johann von Dietrich, kam es 1756 zur Zuteilung von Hofstellen, Land- und Gartenparzellen in Großpold und zum Bau von 90 Häusern in vier Kategorien zur Ansiedlung der überlebenden, wohlhabenden „Transmigranten“. ${ }^{75}$ Von den rund 3000 zwangsdeportierten Menschen waren allerdings nur noch rund 2000 am Leben.

Schließlich folgten 1773 bis 1775 in einer dritten Zeitspanne vier Transporte aus der Steiermark (hauptsächlich aus der Pfarre Stadl) mit 188 Personen, die in Siebenbürgen angesiedelt wurden. Auch aus dem oberösterreichischen Hausruckviertel waren die sich offen $\mathrm{zu}$ ihrer religiösen Überzeugung bekennenden Evangelischen zur Deportation bestimmt worden. ${ }^{76}$

Böhlau Verlag, 2002), 23-80 (53f); Ulrich A. Wien, „Zuwanderung der Landler nach Siebenbürgen“, in Großpold. Ein Dorf in Siebenbürgen, hg. von Martin Bottesch und Ulrich A. Wien (Dößel: Stekovics, 2011), 66-73 (67-71).

74 Heimo Begusch, „Der Geheimprotestantismus“, in Kirchengeschichte der Steiermark, hg. von Karl Anton und Maximilian Liebmann (Graz: Styria Verlag, 1993), 206-19 (215).

75 Buchinger, Hutterer (wie Anm. 68), 57. In Broos (rum. Orăştie) wurden knapp 40 Häuser erworben oder gebaut.

76 Andreas Hochmeir, „Geheimprotestantismus im Land ob der Enns“ in Geheimprotestantismus und evangelische Kirchen in der Habsburgermonarchie und im Erzstift Salzburg (17./18. Jahrhundert), hg. von Rudolf Leeb, Martin Scheutz, und Dietmar Weikl, vol. 51, Veröffentlichungen des Instituts für Österreichische Geschichtsforschung (München: Böhlau, 2009), 155-84 (163f). 
In drei kompakten Siedlungen, in den sogenannten Landler-Dörfern (Neppendorf, Großau, Großpold), konnte die durch mehrfache Differenzen sich abgrenzende Gruppe bedingt durch wechselseitig sich verstärkende Faktoren bis zur Auswanderung am Ende des 20. Jahrhunderts ihre Identität erhalten.

\section{Zusammenfassung}

Verschiedene Aspekte des in Relation zur reformatorischen Theologie stehenden Refugiums Siebenbürgen konnten benannt werden. Unter der Vorherrschaft der Osmanen wurden (1) durch einen politischen Exilanten (Johannes Honterus) auf seinen Exilstationen reformatorische Einflüsse rezipiert, nach Siebenbürgen importiert und mit einiger Verzögerung als humanistisch geprägte Stadtreformation realisiert. (2) Der nach Siebenbürgen zwangsmigrierte und rasch zum ersten Superintendenten erkorene Paul Wiener stabilisierte die Wittenbergisch orientierte Reformation. (3) Unstete kontroverstheologisch agitierende Wanderer wie Francesco Stancaro - bereiteten den Boden für die nachfolgende Zuwanderungswelle. (4) Der Antitrinitarier Giorgio Biandrata strebte mit Verbündeten letztlich vergeblich - danach, in der Ständemonarchie Siebenbürgens eine unitarische „Staatsreligion“ zu verankern. Unterstützt durch dissimulierende Landtagsbeschlüsse (1564/1568) entwickelte sich Siebenbürgen daher zur Pionierregion der Religionsfreiheit, in der die aus der Reformation entstandenen Glaubensgemeinschaften politisch anerkannt wurden. Siebenbürgen wurde vorübergehend zum Refugium devianter, vornehmlich antitrinitarisch gesinnter Flüchtlinge aus ganz Europa. Das vom Landtag 1595 schließlich sanktionierte System der vier rezipierten Religionen (röm.-katholisch, lutherisch, calvinistisch, unitarisch) einschließlich der mehr oder weniger tolerierten ostkirchlichen Orthodoxie galt als Grundgesetz im Fürstentum Siebenbürgen. Das mussten selbst die Habsburger nach der Rückeroberung im Diploma Leopoldinum formal anerkennen. (5) Während des 30-jährigen Krieges lehrten an der 1622 begründeten Reformierten Akademie in Weißenburg renommierte, aus Zentraleuropa geflüchtete Universitätslehrer. (6) Im selben Zeitraum erfolgte die Aufnahme der vertriebenen Täufergemeinden der Hutterer in Siebenbürgen (besonders in Unterwinz), die nicht zuletzt durch Zuwachs von sog. „Transmigranten“ im 18. Jahrhundert nicht ausstarben.

Die religionsrechtlichen Grundbedingungen in der Pionierregion der Religionsfreiheit nutzten die Habsburger Karl VI. und Maria Theresia, um - auch nach dem Ende der osmanischen Präponderanz in der Region Siebenbürgen diese Sonderkultur für ihre absolutistische Regentschaft $\mathrm{zu}$ nutzen. Um die 
religiöse, römisch-katholische Homogenität innerhalb des Kernterritoriums zu erzwingen, wurden großflächig Geheimprotestanten in Oberösterreich, Steiermark und Kärnten aufgespürt und durch Zwangsmigration innerhalb des Habsburger Imperiums ohne Rücksicht auf Verluste umgesiedelt. Trotz der anfänglichen Mortalität von etwa 30\% konnten diese Gruppen von Transmigranten in drei Ortschaften eine eigene Identität entwickeln, bewahren und zugleich die durch die Wirkungen der Reformation als ethnisch-religiöse Sondergruppe der evangelischen „Sachsen“ Siebenbürgens profiliert stärken.

\section{Resultate und Perspektiven}

Die politische und religiöse Sonderstellung und -entwicklung Siebenbürgens im Spätmittelalter prädisponierte die Region zum Laboratorium für religiöse Heterogenität. Die spezifischen regionalen, heterogenen Rechtsräume beförderten vor dem Hintergrund humanistischer Grundhaltungen die Entfaltung zum Teil diffuser, mindestens aber nicht stringent an festen konfessionellen Konturen ausgerichteter Religionskulturen.

Gepaart mit politisch-ökonomischem Pragmatismus und politisch eingehegt durch die Präponderanz der Osmanen (1540-1690) entstand innerhalb der nicht zu idealisierenden - Pionierregion der Religionsfreiheit ein „Fluchtraum“, der sich - trotz zeitweiliger Parallelen in Ostmitteleuropa - schließlich als einzige Region dauerhaft als normgebundenes, plurikonfessionelles Gemeinwesen nicht zuletzt durch den Zuzug von Religionsflüchtlingen stabilisierte.

$\mathrm{Zu}$ erforschen bleiben die Wechselwirkungen zwischen Istanbul und Siebenbürgen, die wechselnden Motive und Strategien der heteronomen Stände/nationes sowie die Frage, ob das nicht-absolutistische Regierungsmodell Siebenbürgens diese Konstellationen begünstigt haben. 